\title{
Leptin and Leptin-to-Adiponectin Ratio Predict Adiposity Gain in Nonobese Children over a Six-Year Period
}

\author{
Meixian Zhang, MD, PhD,' Hong Cheng, BSc,' Xiaoyuan Zhao, BSc,' Dongqing Hou, MSc, \\ Yinkun Yan, PhD,' Katherine Cianflone, PhD, Ming Li, PhD, and Jie Mi, MD, PhD'
}

\section{Abstract}

Background: Previous longitudinal studies have shown inconsistent results regarding the influence of adipokines on changes in weight and body fat. We aimed to determine the predictive value of serum leptin, adiponectin, and their ratio on subsequent changes in obesity measures in children.

Methods: Two hundred forty-six obese and 532 nonobese children aged 6-11 years were remeasured for BMI and waist circumference after $6.4 \pm 0.2$ years. $Z$-score of BMI was used to standardize for age and sex. Obesity was defined using the international BMI cutoffs. Waist-to-height ratio (WHtR) was calculated to define central obesity using a boundary value of 0.5 . Fasting serum leptin and adiponectin levels were measured at baseline.

Results: Newly identified obese children had significantly higher levels of leptin and leptin-to-adiponectin ratio than nonobese children. There were lower adiponectin levels in boys with persistent obesity versus those with transient obesity. After adjusting for age, Tanner stage, and corresponding adiposity measures at baseline, leptin levels and leptin-to-adiponectin ratio were positively associated with BMI Z-score gain in girls and WHtR gain in boys. An inverse association between leptin and BMI Z-score gain was detected in boys. Stratified analyses revealed significant associations only in the nonobese and prepubertal group. There were no significant associations between adiponectin and changes in obesity measures.

Conclusions: High leptin levels and leptin-to-adiponectin ratio are sex-specific predictors of obesity measures gain in nonobese and prepubertal children. Body composition measurement is necessary to assess fat mass growth and distribution during childhood and adolescence.

Keywords: leptin, obesity, adiposity gain, prediction, prospective study, childhood

\section{Introduction}

$\mathbf{T}$ he increasing prevalence of obesity is one of the most important current health problems not only in adulthood but also in childhood. Obesity is a known risk factor for the development of cardiovascular disease, type 2 diabetes, dyslipidemia, hypertension, and some cancers, even in the young. Chinese people are more likely to develop central obesity, which has been associated with a higher risk of developing cardiovascular disease. ${ }^{1}$

Although several obesity treatment programs are available for adults, most of them are not very successful based on their unproven long-term efficacy. There is a growing body of evidence showing that obese children respond better than obese adolescents and adults to lifestyle interventions. ${ }^{2}$ In addition, past epidemiologic literature that tracked obesity showed that children may naturally reverse

\footnotetext{
'Department of Epidemiology, Capital Institute of Paediatrics, Beijing, P.R. China.

${ }^{2}$ Centre de Recherche de l'Institut Universitaire de Cardiologie \& Pneumologie de Québec (CRIUCPQ), Université Laval, Québec, Canada.

${ }^{3}$ Key Laboratory of Endocrinology, Department of Endocrinology, Ministry of Health, Peking Union Medical College Hospital, Chinese Academy of Medical Sciences and Peking Union Medical College (CAMS \& PUMC), Beijing, P.R. China.
}

(c) Meixian Zhang et al., 2017; Published by Mary Ann Liebert, Inc. This Open Access article is distributed under the terms of the Creative Commons Attribution Noncommercial License (http://creativecommons.org/licenses/by-nc/4.0/) which permits any noncommercial use, distribution, and reproduction in any medium, provided the original author(s) and the source are credited. 
overweight in adolescence during a rapid period of growth, while about half of obese school-age children become obese as adults. ${ }^{3}$ Thus, the prevention, as well as treatment of overweight and obesity in adulthood, necessitates the detection of children who are likely to become obese as adults.

Adipokines, which are produced by adipose tissue, are a link between obesity and its related complications and have been recommended as adipose tissue biomarkers. Leptin is a major adipokine that regulates weight balance and energy homeostasis. ${ }^{4}$ Adiponectin is a protein that is secreted exclusively by adipose tissue and improves insulin sensitivity, thereby regulating lipid and glucose metabolism. ${ }^{5}$ In our previous study, we conducted a crosssectional analysis of a large population of Chinese children and adolescents, and the results suggested that serum leptin, adiponectin, and the leptin-to-adiponectin ratio were informative biomarkers for obesity, central obesity, metabolic syndrome, and abnormal metabolic profile even in normal weight children. ${ }^{6}$ Recent studies demonstrate that obesity across the life span has a cumulative influence on adipokines. ${ }^{7,8}$ However, there were differential associations of adipokines with adiposity at different life stages. In addition, in previous longitudinal studies conducted in children, the results of the influence of adipokines on changes in weight and body fat have been inconsistent. ${ }^{9-12}$ Therefore, we conducted a prospective study on Chinese primary school students to test the hypothesis that hyperleptinemia, hypoadiponectinemia, and a high leptin-to-adiponectin ratio may be predictive of subsequent weight gain and development of obesity in baseline obese and nonobese children.

\section{Subjects and Methods}

\section{Subjects}

Subjects were recruited from the Beijing Child and Adolescent Metabolic Syndrome Study (BCAMS), which was a cross-sectional school-based survey initiated in 2004 in Beijing, China. The study sample and data collection have been described elsewhere. ${ }^{13}$ To explore change in obesity status from childhood to adolescence, we conducted a follow-up study in a young subcohort of children aged 611 years. In total, 1626 children aged 6-11 years had blood samples and underwent a baseline examination in 2004. For longitudinal analysis, 848 individuals who did not attend the follow-up examination at the six-year interval in December 2010 were excluded from the participant pool. The remaining 778 participants, including 246 obese $(58.6 \%$ of those eligible) and 532 nonobese children (44.1\% of those eligible), were eligible for analysis. They were reassessed for BMI and waist circumference (WC) using the same standard protocol as measured at baseline. Overweight and obesity were defined using the sex- and age-specific BMI cutoffs recommended by the International Obesity Task Force. ${ }^{14}$ Written informed consent was obtained from all subjects and from their parents or guardians. The study was approved by the Ethics Committee and Institutional Review Board of Capital Institute of Pediatrics.

\section{Measurement of Anthropometric Parameters}

Anthropometric data were collected by trained health technicians using a standard protocol. All instruments were validated following the standard methods of the manufacturers. ${ }^{15}$ Weight was measured to the nearest $0.1 \mathrm{~kg}$ on a balance beam scale, and height was measured to the nearest $0.1 \mathrm{~cm}$ with a wall-mounted stadiometer. WC was measured midway between the lowest rib and the superior border of the iliac crest after normal expiration with an inelastic measuring tape to the nearest $0.1 \mathrm{~cm}$. BMI was calculated as weight in kilograms divided by the square of height in meters. Waist-to-height ratio (WHtR) was calculated to define central obesity using a boundary value of $0.5{ }^{16}$ Pubertal developmental stage was determined based on breast development in girls and testicular volume in boys by trained physicians, according to the criteria described by Marshall and Tanner. ${ }^{17}$ Prepuberty (Tanner I) and puberty (Tanner II or later) were classified according to onset of puberty.

\section{Leptin and Adiponectin Measurement}

At baseline, blood samples were collected from an antecubital vein in vacutainer tubes after a 12-hour overnight fast. Within 30 minutes, plasma and serum aliquots were separated and stored at $-80^{\circ} \mathrm{C}$ until further analysis. All antibodies, reagents, and the adiponectin standard were purchased from Phoenix Pharmaceuticals, Inc. (Belmont, CA) Serum adiponectin was measured by ELISA with an intra-assay and interassay coefficient of variation $(\mathrm{CV})$ of $<5.4 \%$ and $<8.5 \%$, respectively, while serum leptin was measured by ELISA with an intra-assay and interassay CV of $<7.4 \%$ and $<9.3 \%$, respectively. ${ }^{6,18}$

\section{Statistical Analyses}

The analyses were conducted separately in boys and girls. The results are expressed as the mean \pm standard deviation (SD) or geometric means $(95 \%$ confidence interval) for continuous variables and numbers (proportions) for categorical variables. The BMI was standardized to age- and gender-specific $Z$-score, which indicates the distance of a particular value from the mean in units of SD as follows: [(observed value - mean)/SD], based on national reference data for China. ${ }^{19}$ The adipokines had skewed distributions and were natural logarithm transformed for analysis. The mean values for each variable were compared between boys and girls with Student's $t$-test or analysis of covariance (ANCOVA), adjusting for baseline age and pubertal stage. Proportions of sex, Tanner stage, and obesity were compared using $\chi^{2}$ test. Multivariate linear regression models were used to identify the associations between adipokines and changes in adiposity measures. Age, Tanner stage, and adiposity indices at baseline were adjusted for as covariates. All statistical analyses were performed with Statistical Package for Social Sciences (SPSS) version 20.0 software for Windows (SPSS, Inc., Chicago, IL). Two-sided $p$ values $<0.05$ were considered statistically significant. 


\section{Results}

A total of 778 subjects $(47.8 \%)$ were followed for $6.4 \pm 0.2$ years. Comparisons of the baseline characteristics of the subjects between those included and those excluded are displayed in Table 1 . The participants included were younger and heavier with higher levels of adiposity measurements (BMI, BMI Z-score, and WHtR) and a higher prevalence of obesity and central obesity than those lost to follow-up.

\section{Characteristics of Participants at Baseline and Follow-Up}

General demographic, anthropometric, and marker characteristics of the participants at baseline and follow-up are presented in Table 2. There were no differences in age between the two genders, but girls had an advanced pubertal stage. All the adiposity measurements (BMI, BMI $Z$-score, WC, and WHtR) and the prevalence of obesity and central obesity at both surveys were significantly higher in boys than in girls. After adjusting for age and Tanner stage, no differences in baseline leptin, adiponectin, or their ratio were detected between boys and girls.

During the follow-up period, 58 children ( 27 boys and 31 girls) were newly identified as obese, with an incidence of obesity of $10.9 \%$ (58/532). At the second survey, $64.2 \%$ (158/246) of previously obese children remained obese as they aged into adolescence. The persistence of obesity

\begin{tabular}{|c|c|c|c|c|}
\hline Characteristics & $\begin{array}{c}\text { Participants } \\
(n=778)\end{array}$ & $\begin{array}{l}\text { Lost to } \\
\text { follow-up } \\
(n=848)\end{array}$ & $\chi^{2} / t$ & $p$ \\
\hline Boys (\%) & 54.1 & 54.2 & 0.003 & 0.957 \\
\hline Age (years) & $9.4 \pm 1.5$ & $9.8 \pm 1.6$ & -5.947 & $<0.001$ \\
\hline BMI $\left(\mathrm{kg} / \mathrm{m}^{2}\right)$ & $20.6 \pm 4.4$ & $20.1 \pm 4.5$ & 2.175 & 0.030 \\
\hline BMI Z-score & $1.4 I \pm 1.51$ & $1.16 \pm 1.50$ & 3.354 & 0.001 \\
\hline$W C(\mathrm{~cm})$ & $68.3 \pm 12.1$ & $67.4 \pm 12.0$ & 1.605 & 0.109 \\
\hline WHtR & $0.49 \pm 0.07$ & $0.48 \pm 0.07$ & 4.148 & $<0.001$ \\
\hline Obesity $^{\mathrm{a}}(\%)$ & 31.6 & 20.5 & 26.097 & $<0.001$ \\
\hline Central obesity ${ }^{\mathrm{b}}(\%)$ & 46.8 & 35.4 & 22.073 & $<0.001$ \\
\hline
\end{tabular}

Data are expressed as mean $\pm S D$ or frequency, and differences between those included and those excluded were examined using Student's $t$-test or $\chi^{2}$ test.

${ }^{a}$ Obesity was defined using the age- and sex-specific BMI cutoff points recommended by the International Obesity Task Force.

${ }^{b}$ Central obesity was defined as $\mathrm{WH} t \mathrm{R} \geq 0.50$.

$\mathrm{SD}$, standard deviation; WC, waist circumference; WHtR, waist-to-height ratio.
$(69.2 \%$ vs. $52.7 \%, p<0.05)$ but not the incidence of obesity $(10.8 \%$ vs. $11.0 \%, p>0.05)$ was significantly higher in boys than in girls.

\section{Baseline Adipokine Levels in Children with New-Onset or Persistent Obesity}

Over the follow-up period, the newly identified obese children had higher leptin levels and leptin-to-adiponectin ratios than sustained nonobese children, regardless of gender (all $p<0.001$ ), after adjusting for age and Tanner stage (Table 3 ). The above differences were not significant after further adjusting for basal BMI. Within the baseline obese group, serum adiponectin was significantly decreased in boys with persistent obesity versus with transient obesity (9.8 vs. $12.5 \mathrm{mg} / \mathrm{L}, p=0.012$ ), but the difference was not noted in girls. The comparisons of leptin, adiponectin, and leptin-to-adiponectin ratio were replicated when WHtR was used to define central obesity (data not shown).

\section{Prediction of Weight Gain by Baseline Adipokines across Obesity Categories}

Leptin, adiponectin, and their ratio were significantly correlated with the adiposity variables, including BMI, BMI Z-score, and WHtR, measured at baseline irrespective of pubertal stage, and the correlation was stronger in the nonoverweight group than that in the overweight or obese group (Supplementary Table S1; Supplementary Data are available online at www.liebertpub.com/chi). Changes in adiposity measures over the six-year follow-up were then calculated to express weight gain. As shown in Table 4, high leptin levels predicted a greater BMI $Z$-score gain in girls but a lower BMI $Z$-score gain in boys. Stratified analyses by obesity status revealed significant associations of leptin and the leptin-to-adiponectin ratio with changes in BMI Z-score only in the nonobese group.

Then, we further divided the nonobese children into nonoverweight and overweight groups, and the inverse association between leptin levels and BMI $Z$-score gain in boys was only in the overweight group (Supplementary Table S2). We divided the obese group in tertiles to clarify different trends that might be present across obesity degrees. We found that there was an increase relationship trend between serum leptin and BMI Z-score gain across obesity degrees, although not significant (Supplementary Table S2).

Leptin was positively associated with an increase in WHtR in boys (Table 5). Stratified analyses revealed a significant association in boys only in the noncentrally obese group. The predictive ability of the leptin-toadiponectin ratio on change in WHtR was similar to that of leptin. There were no associations between serum adiponectin and changes in any adiposity measures.

\section{Puberty Modified the Predictive Value of Baseline Adipokines for Weight Gain in Nonobese Children}

Further analyses stratified by puberty indicated that the association between the leptin-to-adiponectin ratio and 
Table 2. Participant Characteristics at Baseline and Follow-Up

\begin{tabular}{|c|c|c|c|c|c|c|}
\hline \multirow[b]{2}{*}{ Variables } & \multicolumn{3}{|c|}{ At baseline } & \multicolumn{3}{|c|}{ At follow-up ( $6.4 \pm 0.2$ years later) } \\
\hline & All $(n=778)$ & Boys $(n=421)$ & Girls $(n=357)$ & All $(n=778)$ & Boys $(n=421)$ & Girls $(n=357)$ \\
\hline Age (years) & $9.4 \pm 1.5$ & $9.4 \pm 1.5$ & $9.3 \pm 1.6$ & $15.8 \pm 1.5$ & $15.9 \pm 1.5$ & $15.8 \pm 1.6$ \\
\hline \multicolumn{7}{|l|}{ Tanner stage $[n(\%)]$} \\
\hline I & $509(67.9)$ & $322(80.1)$ & $187(53.7)$ & l & l & l \\
\hline 2 & 157(20.9) & $63(15.7)$ & $94(27.0)$ & l & l & I \\
\hline $3-5$ & $84(11.2)$ & $17(4.2)$ & $67(19.3)^{* *}$ & I & l & l \\
\hline Height $(\mathrm{cm})$ & $139.2 \pm 10.9$ & $139.9 \pm 10.7$ & $138.4 \pm 1 \mid .2$ & $168.4 \pm 8.7$ & $173.5 \pm 7.2$ & $162.4 \pm 6.0 * *$ \\
\hline Weight (kg) & $40.9 \pm 13.0$ & $42.8 \pm 13.4$ & $38.5 \pm 12.0 * *$ & $72.4 \pm 18.7$ & $79.0 \pm 18.7$ & $64.5 \pm 15.3^{* * *}$ \\
\hline $\mathrm{BMI}\left(\mathrm{kg} / \mathrm{m}^{2}\right)$ & $20.6 \pm 4.4$ & $21.4 \pm 4.5$ & $19.7 \pm 4.0 * *$ & $25.3 \pm 5.5$ & $26.1 \pm 5.6$ & $24.4 \pm 5.2 * *$ \\
\hline BMI Z-score & $|.4| \pm|.5|$ & $1.68 \pm 1.59$ & $1.09 \pm 1.35^{* *}$ & $1.58 \pm 1.66$ & $1.70 \pm 1.66$ & $1.43 \pm 1.65^{*}$ \\
\hline WC $(\mathrm{cm})$ & $68.3 \pm 12.1$ & $71.2 \pm 12.5$ & $65.0 \pm 10.8^{* *}$ & $81.8 \pm 14.1$ & $86.1 \pm 14.2$ & $76.7 \pm 12.2^{* *}$ \\
\hline WHtR & $0.489 \pm 0.069$ & $0.507 \pm 0.070$ & $0.469 \pm 0.06 I^{* *}$ & $0.485 \pm 0.076$ & $0.496 \pm 0.078$ & $0.472 \pm 0.07 \mid * *$ \\
\hline Obesity [n (\%)] & $246(31.6)$ & $172(40.9)$ & $74(20.7)^{* *}$ & $216(27.8)$ & $146(34.7)$ & $70(19.6)^{* *}$ \\
\hline Central obesity [n (\%)] & $364(46.8)$ & $247(58.8)$ & $117(32.8)^{* *}$ & $307(39.5)$ & $20 \mathrm{I}(47.7)$ & $106(29.7)^{* *}$ \\
\hline \multicolumn{7}{|c|}{ Changes in adiposity measures } \\
\hline$\Delta$ weight $(\mathrm{kg})$ & l & I & l & $31.5 \pm 13.3$ & $36.2 \pm 12.3$ & $26.0 \pm 12.2^{* *}$ \\
\hline$\Delta \mathrm{BMI}\left(\mathrm{kg} / \mathrm{m}^{2}\right)$ & l & l & l & $4.7 \pm 3.5$ & $4.7 \pm 3.4$ & $4.7 \pm 3.6$ \\
\hline$\Delta \mathrm{BMI} Z$-score & l & I & l & $0.17 \pm 1.20$ & $0.02 \pm 1.11$ & $0.34 \pm 1.28 * *$ \\
\hline$\Delta W C(\mathrm{~cm})$ & l & l & l & $13.5 \pm 10.0$ & $15.0 \pm 10.5$ & $11.7 \pm 9.1 * *$ \\
\hline$\Delta \mathrm{WHtR}$ & l & I & l & $-0.004 \pm 0.056$ & $-0.011 \pm 0.059$ & $0.003 \pm 0.05 I^{* *}$ \\
\hline Leptin $(\mathrm{ng} / \mathrm{mL})^{\mathrm{a}}$ & $4.9(4.4-5.4)$ & $5.0(4.3-5.7)$ & $4.5(3.9-5.3)$ & l & l & l \\
\hline Adiponectin $(\mu \mathrm{g} / \mathrm{mL})^{\mathrm{a}}$ & $12.7(12.2-13.2)$ & $12.8(12.1-13.5)$ & $12.6(11.8-13.4)$ & I & l & l \\
\hline Leptin/adiponectin $^{\mathrm{a}}$ & $0.39(0.35-0.44)$ & $0.39(0.33-0.46)$ & $0.36(0.30-0.43)$ & l & l & l \\
\hline
\end{tabular}

Values are expressed as the mean \pm SD or $n(\%)$. Obesity was defined based on the International Obesity Task Force BMI cutoffs. Central obesity was defined by $\mathrm{WHtR} \geq 0.50$.

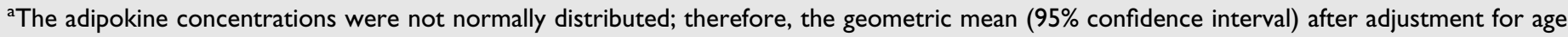
and Tanner stage is shown.

${ }^{*} p<0.05,{ }^{*} p<0.001$, significant difference between boys and girls.

$\Delta$, value at follow-up - value at baseline; /, not available.

BMI Z-score gain in nonobese children was significant only in the prepubertal subgroup (boys: $\beta=-0.155$, $p=0.034$; girls: $\beta=0.251, p=0.006$ ), not in the pubertal subgroup. In noncentrally obese boys, high levels of leptin $(\beta=0.019, p<0.001)$ and the leptin-to-adiponectin ratio $(\beta=0.016, p<0.001)$ were also positively associated with a greater increase in WHtR in the prepubertal subgroup, but the association for adiponectin was in the pubertal subgroup $(\beta=0.026, p=0.045)$.

\section{Discussion}

The current study provides longitudinal information about the predictive value of adipokine markers for weight gain and the development of obesity in both baseline obese and nonobese children. Our results showed that relatively high serum leptin concentrations and leptin-to-adiponectin ratio were positively associated with BMI Z-score gain in baseline nonobese girls and with WHtR gain in noncentrally obese boys over the six-year follow-up period. Our findings confirm that sex-dependent associations exist between baseline leptin levels and subsequent changes in adiposity measures in children and support the consideration of baseline obesity and pubertal status as potential confounders.

Several previous longitudinal studies found inverse relationships between leptin and adiposity changes in nonobese young adults ${ }^{20,21}$ and children. ${ }^{22,23}$ It is worth noting 
Table 3. Comparison of Baseline Adipokine Levels between Obese and Nonobese Children at Follow-Up Stratified by Sex and Baseline Obesity Status

\begin{tabular}{|c|c|c|c|c|c|c|c|}
\hline \multirow[b]{2}{*}{ Sex } & \multicolumn{2}{|c|}{ Obesity } & \multirow[b]{2}{*}{$n(\%)$} & \multirow[b]{2}{*}{ BMI Z-score } & \multirow[b]{2}{*}{ Leptin (ng/mL) } & \multirow[b]{2}{*}{ Adiponectin $(\mu \mathrm{g} / \mathrm{mL})$} & \multirow[b]{2}{*}{ Leptin/adiponectin } \\
\hline & Baseline & Follow-up & & & & & \\
\hline$A l^{a}$ & - & - & $474(89.1)$ & $0.50 \pm 0.04$ & $2.6(2.3-2.9)$ & $14.0(13.3-14.7)$ & $0.19(0.16-0.21)$ \\
\hline \multirow[t]{5}{*}{$(n=778)$} & - & + & $58(10.9)$ & $1.52 \pm 0.13$ & $7.6(5.5-10.5)$ & $13.5(\mid 1.8-15.5)$ & $0.58(0.40-0.83)$ \\
\hline & \multicolumn{2}{|c|}{$p$} & & $<0.001$ & $<0.001$ & 0.640 & $<0.001$ \\
\hline & + & - & $88(35.8)$ & $2.84 \pm 0.10$ & $12.5(10.5-14.8)$ & $11.4(10.0-12.9)$ & I.II (0.89-I.37) \\
\hline & + & + & $158(64.2)$ & $3.25 \pm 0.07$ & $14.1(12.5-16.0)$ & $9.9(9.0-10.8)$ & $1.43(1.23-1.67)$ \\
\hline & \multicolumn{2}{|c|}{$p$} & & 0.001 & 0.249 & 0.076 & 0.060 \\
\hline Boys & - & - & $222(89.2)$ & $0.47 \pm 0.07$ & $2.2(1.8-2.6)$ & 14.9 (|4.0-15.9) & $0.15(0.12-0.18)$ \\
\hline \multirow[t]{5}{*}{$(n=42 I)$} & - & + & $27(10.8)$ & $1.77 \pm 0.20$ & $7.0(4.1-|| .8)$ & $14.9(12.5-17.8)$ & $0.49(0.27-0.87)$ \\
\hline & \multicolumn{2}{|c|}{$p$} & & $<0.001$ & $<0.001$ & 0.989 & $<0.001$ \\
\hline & + & - & $53(30.8)$ & $2.87 \pm 0.11$ & $12.1(9.6-15.1)$ & $12.5(10.7-14.7)$ & $0.97(0.73-1.29)$ \\
\hline & + & + & $119(69.2)$ & $3.36 \pm 0.07$ & $12.9(11.2-14.9)$ & $9.8(8.9-10.9)$ & I.32 (I.II-I.58) \\
\hline & \multicolumn{2}{|c|}{$p$} & & $<0.001$ & 0.602 & 0.012 & 0.077 \\
\hline Girls & - & - & $252(89.0)$ & $0.52 \pm 0.06$ & $3.0(2.6-3.5)$ & $13.2(12.3-\mid 4.2)$ & $0.23(0.19-0.27)$ \\
\hline \multirow[t]{5}{*}{$(n=357)$} & - & + & $31(11.0)$ & $1.30 \pm 0.16$ & $7.8(5.2-11.8)$ & $12.3(10.1-15.1)$ & $0.64(0.39-1.04)$ \\
\hline & \multicolumn{2}{|c|}{$p$} & & $<0.001$ & $<0.001$ & 0.510 & $<0.001$ \\
\hline & + & - & $35(47.3)$ & $2.74 \pm 0.20$ & I3.8 (10.8-17.7) & $9.8(8.0-11.9)$ & $1.43(1.03-1.98)$ \\
\hline & + & + & 39 (52.7) & $3.05 \pm 0.19$ & $17.8(14.0-22.5)$ & $10.3(8.5-12.5)$ & 1.73 (1.27-2.37) \\
\hline & \multicolumn{2}{|c|}{$p$} & & 0.265 & 0.146 & 0.717 & 0.398 \\
\hline
\end{tabular}

Values are expressed as the mean \pm SE or geometric mean ( $95 \%$ confidence interval) after adjustment for age and Tanner stage. Obesity was defined based on the International Obesity Task Force BMI cutoffs.

Bold indicates that the associations showed statistical significance.

"-" indicates nonobese state and "+" indicates obese state.

${ }^{a}$ Additionally controlled for sex.

SE, standard error.

Table 4. Regression Coefficients $(\beta)$ and Standard Error of Natural Logarithm of Each Adipokine on Change in BMI Z-Score Stratified by Sex and Obesity Status at Baseline

\begin{tabular}{|c|c|c|c|c|c|c|c|c|}
\hline \multirow[b]{2}{*}{ Sex } & \multirow{2}{*}{$\begin{array}{c}\text { Obesity } \\
\text { status }\end{array}$} & \multirow[b]{2}{*}{$n$} & \multicolumn{2}{|c|}{ Leptin $^{a}$} & \multicolumn{2}{|c|}{ Adiponectin $^{\mathbf{a}}$} & \multicolumn{2}{|c|}{ Leptin/adiponectin ${ }^{a}$} \\
\hline & & & $\beta$ (SE) & $p$ & $\beta$ (SE) & $p$ & $\beta$ (SE) & $p$ \\
\hline \multirow[t]{3}{*}{ Boys } & All & 421 & $-0.148(0.065)$ & 0.022 & $-0.135(0.102)$ & 0.183 & $-0.063(0.054)$ & 0.245 \\
\hline & Nonobese & 249 & $-0.131(0.072)$ & 0.070 & $0.125(0.120)$ & 0.298 & $-0.128(0.061)$ & 0.036 \\
\hline & Obese & 172 & $-0.136(0.134)$ & 0.312 & $-0.332(0.175)$ & 0.060 & $0.033(0.105)$ & 0.753 \\
\hline \multirow[t]{3}{*}{ Girls } & All & 357 & $0.312(0.075)$ & $<0.001$ & $-0.097(0.117)$ & 0.406 & $0.224(0.060)$ & $<0.001$ \\
\hline & Nonobese & 283 & $0.209(0.080)$ & 0.009 & $-0.007(0.121)$ & 0.956 & $0.146(0.066)$ & 0.027 \\
\hline & Obese & 74 & $0.169(0.255)$ & 0.509 & $0.159(0.317)$ & 0.618 & $0.023(0.188)$ & 0.905 \\
\hline
\end{tabular}

Bold indicates that the associations showed statistical significance.

${ }^{a}$ Data with skewed distributions were natural logarithm-transformed for analysis.

Adjusted for age, Tanner stage, and BMI Z-score at baseline.

Obesity was defined based on the International Obesity Task Force BMI cutoffs. 


\begin{tabular}{|c|c|c|c|c|c|c|c|c|}
\hline \multirow[b]{2}{*}{ Sex } & \multirow[b]{2}{*}{ Obesity status } & \multirow[b]{2}{*}{$n$} & \multicolumn{2}{|c|}{ Leptin $^{a}$} & \multicolumn{2}{|c|}{ Adiponectin $^{a}$} & \multicolumn{2}{|c|}{ Leptin/adiponectin ${ }^{a}$} \\
\hline & & & $\beta$ (SE) & $p$ & $\beta$ (SE) & $p$ & $\beta$ (SE) & $p$ \\
\hline \multirow[t]{3}{*}{ Boys $^{b}$} & All & 420 & $0.007(0.003)$ & 0.043 & $-0.009(0.005)$ & 0.119 & $0.007(0.003)$ & 0.015 \\
\hline & No central obesity & 173 & $0.015(0.004)$ & $<0.001$ & $-0.004(0.008)$ & 0.614 & $0.011(0.003)$ & 0.001 \\
\hline & Central obesity & 247 & $0.001(0.005)$ & 0.947 & $-0.012(0.008)$ & 0.124 & $0.003(0.004)$ & 0.529 \\
\hline \multirow[t]{3}{*}{ Girls } & All & 357 & $-0.003(0.003)$ & 0.270 & $0.008(0.005)$ & 0.108 & $-0.005(0.003)$ & 0.074 \\
\hline & No central obesity & 240 & $-0.002(0.003)$ & 0.462 & $0.006(0.005)$ & 0.274 & $-0.003(0.003)$ & 0.234 \\
\hline & Central obesity & 117 & $-0.003(0.008)$ & 0.721 & $0.013(0.010)$ & 0.224 & $-0.007(0.006)$ & 0.284 \\
\hline
\end{tabular}

Adjusted for age, Tanner stage, and WHtR at baseline.

Central obesity was defined by $\mathrm{WH} t \mathrm{R} \geq 0.50$.

Bold indicates that the associations showed statistical significance.

a Data with skewed distributions were natural logarithm-transformed for analysis.

baseline WC data were missing in one boy.

that these studies were all conducted with small sample sizes or with a short follow-up interval. In contrast with these studies, the results of most studies were in agreement with ours, showing a positive relationship between circulating leptin levels and greater future BMI or body adiposity..$^{11,12,24}$ Recent studies ${ }^{11,12}$ as well as ours were conducted in the general pediatric East Asian population. In the present study, we first contributed to a growing body of literature by reporting the associations between leptin levels and adiposity variations in Chinese children.

We found that leptin levels sex dependently predicted gains in BMI $Z$-score and WHtR in nonobese children. One explanation for this finding is the gender difference in the direction of the change in fat mass and fat-free mass relationship throughout childhood. BMI is widely used as a surrogate marker of adiposity. The age-related increase in BMI throughout development is driven primarily by a large increase in fat-free mass, particularly in boys. ${ }^{25-27}$ During this period, girls gain more fat mass relative to lean mass, whereas boys experience a prepubertal fat growth followed by rapid gains in fat-free mass and reductions in fat mass. ${ }^{28,29}$

Besides the amount of fat mass, there is a striking sex difference in body fat distribution. Adipose tissue is more likely to be deposited in the abdomen in boys than in girls, ${ }^{30}$ although $\sim 90 \%$ of abdominal fat in children is subcutaneous adipose tissue. ${ }^{31} \mathrm{WC}$ and WHtR are surrogate markers of central obesity and reflect both subcutaneous adipose tissue and visceral adipose tissue. Visceral adipose tissue accumulates more rapidly with age and weight gain in boys than in girls, although visceral adipose tissue secretes less leptin than subcutaneous adipose tissue. ${ }^{32}$ Therefore, changes in WHtR but not BMI may more accurately estimate the change in adiposity in boys, while a change in BMI $Z$-score is a better indicator of fat gain in girls.

In addition, in the stratified analyses, we found the positive association between leptin and BMI Z-score increase in girls with prepubertal stage, but the negative association in boys with pubertal stage. So, the different sex steroid milieu may influence role of leptin on adiposity change between males and females. ${ }^{33}$ Our findings suggest that resistance to leptin may be present in nonobese girls and noncentrally obese boys, in whom it would predict subsequent adiposity gain, but in some boys with the highest leptin levels, leptin can normally suppress weight gain.

However, in obese children, we found no evidence supporting the associations of leptin with weight gain and obesity persistence over time. The highest levels of baseline leptin were observed in children with persistent obesity, followed by those with transient obesity at baseline and then incident obesity during the follow-up period compared to nonobese children at both surveys. This suggests that serum leptin is more important for concurrent obesity than weight gain or future obesity. This finding is consistent with some previous studies in adult Mauritians ${ }^{34}$ and in African American and white young and older adults. ${ }^{35,36}$ However, this relationship has been challenged by other studies finding positive associations, ${ }^{10,37}$ which have been interpreted as evidence that high levels of leptin are indicative of leptin resistance in overweight and obese people and are a contributing factor to weight gain. As leptin levels are closely related to adipose tissue, the relationship between leptin levels and future weight gain during childhood and adolescence could differ depending 
on the degree of fat mass excess and pubertal development. Accordingly, the discrepancies between our results and those of previous studies may be attributed, in part, to differences in average BMI (common obese vs. morbidly obese). The heterogeneity of the study populations in age, ethnic background, family history of obesity and leptin resistance, and of study design, such as differences in the follow-up interval and methods of adjustment for the variability in body composition, may have also contributed to these contradictory results. The potentially confounding effects of acute dietary and physical activity on leptin and associations with weight change have been overlooked in most studies. Thus, increased leptin levels in obese children may not necessarily reflect leptin resistance, and many children appear to remain leptin sensitive at this age. ${ }^{38}$ This suggests that high leptin levels in obese children may be a consequence of an adaptation to obesity.

Adiponectin is an adipocyte-specific hormone that exerts pleiotropic physiological functions by promoting insulin sensitivity, inhibiting cell death, and suppressing inflammation. Serum adiponectin concentrations are lower in individuals with insulin resistance, type 2 diabetes, and obesity in cross-sectional studies. However, there is a lack of studies investigating the prospective effects of hypoadiponectinemia on the development of obesity in humans. Hypoadiponectinemia was not causally associated with increasing adiposity in obese Pima Indians, ${ }^{39}$ elderly white individuals, ${ }^{36,40}$ or overweight Afro-Jamaicans. ${ }^{41}$ In contrast, there was a significant positive relationship between weight change and baseline adiponectin levels in healthy women who did not develop diabetes over a long follow-up period. ${ }^{42}$ In the current study, we found lower adiponectin concentrations in boys with persistent obesity versus those with transient obesity, and a positive association between baseline adiponectin levels and WHtR gain only in noncentrally obese pubertal boys. However, these results need to be further validated because of the relatively small numbers in the subgroup. These prospective data add to the current literature on the predictive effects of baseline adiponectin levels on increasing adiposity in children.

We also examined the impact of the leptin-to-adiponectin ratio, a joint indicator of adipokine levels, on weight gain from childhood to adolescence. Previous studies indicated that the ratio of serum leptin to adiponectin may reflect compromised adipose tissue function and should be a more efficient predictor of obesity and related complications than leptin or adiponectin alone. ${ }^{6,43}$ However, other studies, including a single cohort with large samples of adults from four ethnicities, demonstrated that this ratio was not more strongly associated with adiposity or insulin resistance than leptin or adiponectin. ${ }^{44}$ Our findings provide evidence that the leptin-to-adiponectin ratio is, similar to leptin, related to gains in adiposity measures. In nonobese prepubertal children, the ratio beyond leptin predicts changes in BMI $Z$-score irrespective of gender. The leptin-to-adiponectin ratio may be a good joint marker for future adiposity gain. Serum leptin and adiponectin can be readily measured in clinical practice and these findings may be used to differentiate the "at risk of obesity" state from nonobese in childhood. The children at high risk for obesity are most in need of weight monitoring and early, intensive intervention.

Our study had several strengths. The present study not only provides the longitudinal relationships of leptin, adiponectin, and their ratio with subsequent BMI gain during childhood and adolescence but also examines the predictive effect of adipokines for gain in WHtR, which is an indicator for central obesity. In addition, the detailed analysis with the sizeable sample is another advantage. We investigated the predictive value stratified by sex, baseline obesity state, and pubertal status. Our results provided evidence of the sex-dependent associations of leptin and the leptin-to-adiponectin ratio with gains in adiposity measures only in nonobese or noncentrally obese children. The direct examination of pubertal status is a clear strength of this study. The stratified analysis by puberty indicated that the onset of puberty weakened the associations. However, our results should be considered preliminary. The modification of effects by puberty needs to be further clarified in studies with larger sample sizes.

Nonetheless, some limitations to this study should be noted. First, loss to follow-up is inevitable over a long-term period. More than half of the eligible children were lost to follow-up, and more obese and younger children were detected in those included than in those excluded in the present analyses. Second, although BMI provides a simple, convenient measurement of obesity, it does not distinguish between body fat mass and fat-free mass. WC and WHtR, as measures of central obesity, do not differentiate subcutaneous fat from visceral fat. Body composition measurement will be guaranteed in the future study. Third, data on lifestyle factors (e.g., eating habits and physical activity) may influence the associations between adipokines and weight gain, but they were not collected during the six-year follow-up.

In conclusion, relatively high serum leptin was associated with greater BMI gain in nonobese girls and with waist gain in noncentrally obese boys during a six-year follow-up period. The ratio of leptin to adiponectin was superior to leptin or adiponectin alone in predicting subsequent weight gain in nonobese or noncentrally obese children. We propose that high serum leptin and leptin-toadiponectin ratio can be considered early biomarkers for identifying children at greater risk of future obesity. Low adiponectin can be considered a predictor of the persistence of obesity in boys. We conclude that the predictive ability of adipokines regarding changes in adiposity is variably dependent on gender, baseline obesity status, and pubertal status.

\section{Acknowledgments}

This work is supported by grants from the National Natural Science Foundation of China (81473062, 
81502872), the National Basic Research Program of China (973 Program, 2013CB530605), Beijing Municipal Science and Technology Key Project (D111100000611002), and the Capital Institute of Pediatrics (FX-15-05).

\section{Author Disclosure Statement}

No competing financial interests exist.

\section{References}

1. Thomas GN, Ho SY, Lam KS, et al. Impact of obesity and body fat distribution on cardiovascular risk factors in Hong Kong Chinese. Obes Res 2004;12:1805-1813.

2. Knop C, Singer V, Uysal Y, et al. Extremely obese children respond better than extremely obese adolescents to lifestyle interventions. Pediatr Obes 2015;10:7-14.

3. Deshmukh-Taskar P, Nicklas TA, Morales M, et al. Tracking of overweight status from childhood to young adulthood: The Bogalusa Heart Study. Eur J Clin Nutr 2006;60:48-57.

4. Jéquier E. Leptin signaling, adiposity, and energy balance. Ann N Y Acad Sci 2002;967:379-388.

5. Okamoto Y, Kihara S, Funahashi T, et al. Adiponectin: A key adipocytokine in metabolic syndrome. Clin Sci (Lond) 2006; 110:267-278.

6. Mi J, Munkonda MN, Li M, et al. Adiponectin and leptin metabolic biomarkers in Chinese children and adolescents. $J$ Obes 2010;2010:892081.

7. Li S, Liu R, Arguelles L, et al. Adiposity trajectory and its associations with plasma adipokine levels in children and adolescents-A prospective cohort study. Obesity (Silver Spring) 2016;24:408-416.

8. Murray ET, Hardy R, Hughes A, et al. Overweight across the life course and adipokines, inflammatory and endothelial markers at age 60-64 years: Evidence from the 1946 birth cohort. Int J Obes (Lond) 2015;39:1010-1018.

9. Kettaneh A, Heude B, Romon M, et al. High plasma leptin predicts an increase in subcutaneous adiposity in children and adults. Eur $J$ Clin Nutr 2007;61:719-726.

10. Fleisch AF, Agarwal N, Roberts MD, et al. Influence of serum leptin on weight and body fat growth in children at high risk for adult obesity. J Clin Endocrinol Metab 2007;92:948-954.

11. Nishimura R, Sano H, Matsudaira T, et al. Changes in body mass index, leptin and adiponectin in Japanese children during a threeyear follow-up period: A population-based cohort study. Cardiovasc Diabetol 2009;8:30.

12. Park JE, Choi HJ, Kim IK, et al. Influence of serum leptin levels on future overweight risk in Korean children. Nutr Metab Cardiovasc Dis 2012;22:260-268.

13. Shan XY, Xi B, Cheng $\mathrm{H}$, et al. Prevalence and behavioral risk factors of overweight and obesity among children aged 2-18 in Beijing, China. Int J Pediatr Obes 2010;5:383-389.

14. Cole TJ, Bellizzi MC, Flegal KM, et al. Establishing a standard definition for child overweight and obesity worldwide: International survey. BMJ 2000;320:1240-1243.

15. World Health Organization. Physical Status: The Use and Interpretation of Anthropometry: Report of a WHO Expert Committee. World Health Organization: Geneva, 1995, pp. 1-452 (Tech. Rep. Ser. no. 854).
16. Browning LM, Hsieh SD, Ashwell M. A systematic review of waist-to-height ratio as a screening tool for the prediction of cardiovascular disease and diabetes: $0 \cdot 5$ could be a suitable global boundary value. Nutr Res Rev 2010;23:247-269.

17. Marshall WA, Tanner JM. Puberty in Human Growth, volume 2. New York and London: Plenum Press. 1986, pp. 171-210.

18. Li M, Yin JH, Zhang K, et al. A highly sensitive enzyme-linked immunosorbent assay for measurement of leptin secretion in human adipocytes. Zhong Hua Yi Xue Za Zhi 2008;88:3293-3297.

19. Ministry of Education of the People's Republic of China. Report on the Physical Fitness and Health Surveillance of Chinese School Students. Higher Education Press: Beijing, 2002, pp. 3-46.

20. Allard C, Doyon M, Brown C, et al. Lower leptin levels are associated with higher risk of weight gain over 2 years in healthy young adults. Appl Physiol Nutr Metab 2013;38:280-285.

21. van Rossum CT, Hoebee B, van Baak MA, et al. Genetic variation in the leptin receptor gene, leptin, and weight gain in young Dutch adults. Obes Res 2003;11:377-386.

22. Byrnes SE, Baur LA, Bermingham M, et al. Leptin and total cholesterol are predictors of weight gain in pre-pubertal children. Int J Obes Relat Metab Disord 1999;23:146-150.

23. Ahmed ML, Ong KK, Morrell DJ, et al. Longitudinal study of leptin concentrations during puberty: Sex differences and relationship to changes in body composition. J Clin Endocrinol Metab 1999;84:899-905.

24. Ahmed ML, Ong KK, Watts AP, et al. Elevated leptin levels are associated with excess gains in fat mass in girls, but not boys, with type 1 diabetes: Longitudinal study during adolescence. J Clin Endocrinol Metab 2001;86:1188-1193.

25. Maynard LM, Wisemandle W, Roche AF, et al. Childhood body composition in relation to body mass index. Pediatrics 2001;107: 344-350.

26. Demerath EW, Schubert CM, Maynard LM, et al. Do changes in body mass index percentile reflect changes in body composition in children? Data from the Fels Longitudinal Study. Pediatrics 2006;117:e487-e495.

27. Eissa MA, Dai S, Mihalopoulos NL, et al. Trajectories of fat mass index, fat free-mass index, and waist circumference in children: Project HeartBeat! Am J Prev Med 2009;37:S34-S39.

28. Weber DR, Leonard MB, Zemel BS. Body composition analysis in the pediatric population. Pediatr Endocrinol Rev 2012;10:130 139.

29. Park HW, Yoo HY, Kim CH, et al. Reference values of body composition indices: The Korean National Health and Nutrition Examination Surveys. Yonsei Med J 2015;56:95-102.

30. Blouin K, Boivin A, Tchernof A. Androgens and body fat distribution. J Steroid Biochem Mol Biol 2008;108:272-280.

31. Shen W, Punyanitya M, Silva AM, et al. Sexual dimorphism of adipose tissue distribution across the lifespan: A cross-sectional whole-body magnetic resonance imaging study. Nutr Metab (Lond) 2009;6:17.

32. Van Harmelen V, Reynisdottir S, Eriksson P, et al. Leptin secretion from subcutaneous and visceral adipose tissue in women. Diabetes 1998;47:913-917.

33. da Silva RP, Zampieri TT, Pedroso JA, et al. Leptin resistance is not the primary cause of weight gain associated with reduced sex hormone levels in female mice. Endocrinology 2014;155:42264236.

34. Hodge AM, De Courten MP, Dowse GK, et al. Do leptin levels predict weight gain? A 5-year follow-up study in Mauritius. Obes Res 1998;6:319-325. 
35. Haffner SM, Mykkänen LA, Gonzalez CC, et al. Leptin concentrations do not predict weight gain: The Mexico City Diabetes Study. Int J Obes Relat Metab Disord 1998;22:695-699.

36. Langenberg C, Bergstrom J, Laughlin GA, et al. Ghrelin, adiponectin, and leptin do not predict long-term changes in weight and body mass index in older adults: Longitudinal analysis of the Rancho Bernardo cohort. Am J Epidemiol 2005;162:1189-1197.

37. Savoye M, Dziura J, Castle J, et al. Importance of plasma leptin in predicting future weight gain in obese children: A two-and-a-halfyear longitudinal study. Int J Obes Relat Metab Disord 2002; 26:942-946.

38. Murer SB, Knöpfli BH, Aeberli I, et al. Baseline leptin and leptin reduction predict improvements in metabolic variables and long-term fat loss in obese children and adolescents: A prospective study of an inpatient weight-loss program. Am J Clin Nutr 2011;93:695-702.

39. Vozarova B, Stefan N, Lindsay RS, et al. Low plasma adiponectin concentrations do not predict weight gain in humans. Diabetes 2002;51:2964-2967.

40. Wedick NM, Snijder MB, Dekker JM, et al. Prospective investigation of metabolic characteristics in relation to weight gain in older adults: The Hoorn Study. Obesity (Silver Spring) 2009;17: 1609-1614.

41. Bennett NR, Boyne MS, Cooper RS, et al. Impact of adiponectin and ghrelin on incident glucose intolerance and on weight change. Clin Endocrinol (Oxf) 2009;70:408-414.
42. Hivert MF, Sun Q, Shrader P, et al. Higher adiponectin levels predict greater weight gain in healthy women in the Nurses' Health Study. Obesity (Silver Spring) 2011;19:409-415.

43. Finucane FM, Luan J, Wareham NJ, et al. Correlation of the leptin:adiponectin ratio with measures of insulin resistance in nondiabetic individuals. Diabetologia 2009;52:2345-2349.

44. Rasmussen-Torvik LJ, Wassel CL, Ding J, et al. Associations of body mass index and insulin resistance with leptin, adiponectin, and the leptin-to-adiponectin ratio across ethnic groups: The MultiEthnic Study of Atherosclerosis (MESA). Ann Epidemiol 2012; 22:705-709.

Address correspondence to:

Jie Mi, MD, PhD

Department of Epidemiology Capital Institute of Paediatrics No. 2 Yabao Road Chaoyang District Beijing 100020 P.R. China

E-mail: jiemi@vip.163.com 